\title{
CREATION OF FEMTOSECOND SHEET-LIKE BUNCHES FOR DRIVING OPTICAL-SCALE DIELECTRIC SLAB ACCELERATOR STRUCTURES*
}

\author{
Changbiao Wang, ${ }^{1}$ J. L. Hirshfield, ${ }^{1,2}$ and T. C. Marshall ${ }^{3}$ \\ ${ }^{1}$ Yale University, Beam Physics Laboratory, 272 Whitney Ave., New Haven, CT 06511, USA \\ ${ }^{2}$ Omega-P Inc., 345 Whitney Ave., Suite 100, New Haven CT 06511, USA \\ ${ }^{3}$ Columbia University, Dept. of Applied Physics, New York, NY 10027, USA
}

\begin{abstract}
Optically chopped bunches can be created by placing a beam stop with an off-axis hole following laser-driven cyclotron autoresonance acceleration. For example, a 500 $\mathrm{MeV}$ electron beam driven by a $5 \mathrm{TW} / 10.6$ micron laser can be chopped into bunches with diameter $\sim 50$ microns and length $\sim 1$ micron. It is furthermore shown that the bunches can be elongated in one transverse dimension and compressed in the other using a quadrupole magnet, so as to serve as the driver for an optical-scale dielectric wake field accelerator. Bunch length spreading from space charge is examined to find limits to transport of $\mathrm{pC}$, micron long bunches.
\end{abstract}

\section{INTRODUCTION}

New techniques for accelerating electrons or positrons using lasers promise very high accelerating gradients $(>1$ $\mathrm{GeV} / \mathrm{m})$. We describe here a technique to prepare a train of bunches of electrons at high energy, each of which is approximately $1 \mu \mathrm{m}(3.5 \mathrm{fsec})$ in length, and which can be used to excite small material structures in vacuum so as to provide $\mathrm{GV} / \mathrm{m}$ accelerating fields. Optically-resonant structures can be precisely fabricated using microcircuit techniques. A specific accelerator concept that could benefit by using laser-wavelength scale structures is the dielectric wakefield accelerator [1,2], discussed here.

Wakefield accelerators are generally attractive because no external source of radiation is used to drive the structure itself. The small transverse dimension in these devices permits the build-up of very high accelerating fields, $\sim \mathrm{GV} / \mathrm{m}$; such large fields in a dielectric structure are possible because the tunnelling-ionization breakdown field of the dielectric can become larger than otherwise when exposed to fsec-duration pulses [3]. Furthermore, the planar wake field structure discussed here has the advantages that it can pass more beam charge, store more energy, and have better stability to transverse beam deflection than does a corresponding cylindrical structure.

\section{MAKING FSEC SHEET BUNCHES}

An attractive feature of a 2D planar dielectric structure was discovered in 1997 for a microwave-scale wake field accelerator [2]. It was shown that the structure can be designed so that many TM-like guided modes are excited by short charge bunches, and that the fundamental period

\footnotetext{
*Research supported by the DoE, Division of High Energy Physics.
}

of the modes can be made equal to the period of the bunch train. The combination of short bunch length and high dielectric constant material favours the creation of sharply-localized pulses of longitudinal electric field. Wake fields from one bunch in a train can be superimposed upon the wake fields from prior bunches. Placement of a test bunch one-half period behind the last drive bunch will allow much higher acceleration gradients than with only one bunch. A comprehensive theory of wake fields in cylindrical dielectric structures [1] and analysis of stability for this geometry [4] have been recently published. It turns out that the use of planar geometry, while not practical for microwave accelerator structures, is attractive when one considers the use of micron-long, tall rectangular cross-section sheet-like bunches which could excite a 3D rectangular dielectric wake field structure with $\mu \mathrm{m}$-scale transverse dimension. Figure 1 shows a schematic of a short rectangular electron bunch travelling between two dielectric layers; the narrow transverse dimension $2 a$ of the channel is $\sim 10 \mu \mathrm{m}$, the thickness of each dielectric layer is $b-a \sim 10 \mu \mathrm{m}$, the length is several $\mathrm{cm}$, and the wide transverse dimension $(H)$ is at least ten times the narrow one $(2 a)$.

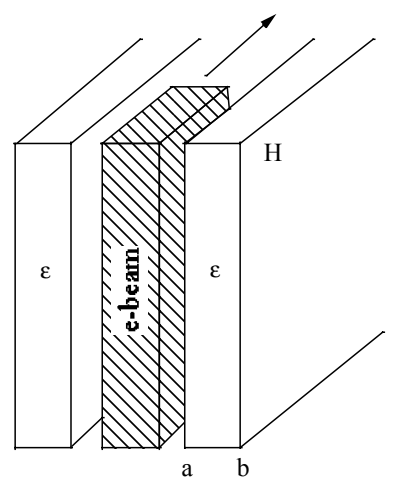

Figure 1: Schematic of bunch travelling through an optical-scale wake field accelerator structure.

A conceptual schematic of a laser-driven cyclotron autoresonance acceleration (LACARA) [5] and other elements needed to form the short bunches is shown in Fig. 2. Noteworthy are: the off-axis beam stop, the beamsqueezing quadrupole, and the dielectric wake field structure. At ATF (BNL), the first LACARA is to be operated as an accelerator; however, for the application of making short, rectangular-profile bunches of micron 
dimensions suitable for wake field acceleration in an optical-scale structure, it is necessary to employ an input bunch to LACARA that has very small emittance. In the following, a beam with normalized emittance of $1 \mathrm{~mm}$ mrad at an energy of $500 \mathrm{MeV}$ was taken [6]; this choice of energy is also significant in staging a sequence of wake field structures, since it fixes the order-of-magnitude of possible energy gain by a test bunch in each stage, and thus the number of stages required to reach a given final energy. The LACARA as employed here is not much of an accelerator, but a phase-buncher. The solenoidal field is taken as $1.76 \mathrm{~T}$ and is $5 \mathrm{~m}$ in length, and a $5 \mathrm{TW} \mathrm{CO}$ Gaussian profile laser beam is introduced along the axis of the solenoid. The bunch charge is taken to be $1 \mathrm{nC}$ with a 1 psec length, and the input bunch radius to LACARA is taken to be $30 \mu \mathrm{m}$.

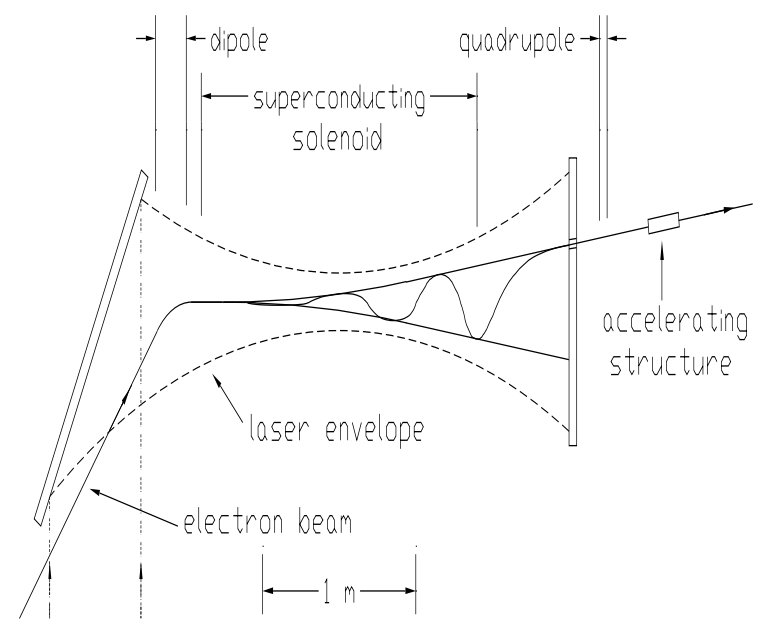

Figure 2: Schematic of LACARA with added beam stop to produce a chopped beam that is formed into sheet-like form using a quadrupole, for generation of intense wake fields in a planar dielectric accelerating structure. Radial scale is expanded for clarity.

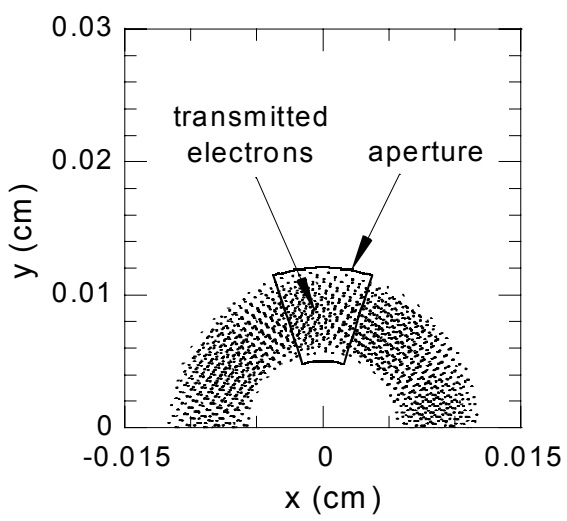

Figure 3: Particle distribution in $x-y$ plane at $z=625 \mathrm{~cm}$ (back of beam stop). 10\% of electrons are transmitted.

The acceleration of this bunch of electrons is quite small, with the relativistic energy factor $\gamma$ changing by only $0.2 \%$; thus only a small fraction of the $5 \mathrm{TW}$ laser pulse energy is used. At the end of the interaction region where the axial field is weak, electrons are distributed around an annular ring. Their motions projected upon the front surface of the beam stop at $z=620 \mathrm{~cm}$ are such that particles have successive loci that advance around the $x-y$ plane at an angular frequency that corresponds to the 35.3 fsec period of the $10.6 \mu \mathrm{m}$ laser wavelength. The beam stop contains a small hole for transmitting a fraction of these electrons, shown at the output end at $z=625 \mathrm{~cm}$ (Fig. 3). The hole transmits an angular section of the beam profile at the beam stop, dimensioned to form a micropulse of about 3.5 fsec in duration ( $10 \%$ of a cycle). Computed current pulses ( 2 out of the train of about 30) are shown in Fig. 4. The transmitted charge in each of the 3.5 fs micro-bunches is $3.5 \mathrm{pC}$, i.e., $2 \times 10^{7}$ electrons.

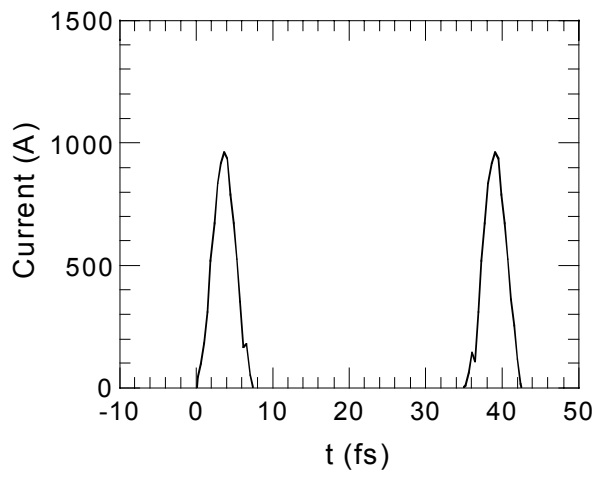

Figure 4: Current transmitted by the beam stop aperture as a function of time at $z=625 \mathrm{~cm}$.

Beyond the beam stop is located a quadrupole element that will prepare a "sheet" profile beam pulse. The quadrupole is located between $z=630$ and $635 \mathrm{~cm}$, and distorts the bunch into a sheet profile at $z \sim 666 \mathrm{~cm}$; the
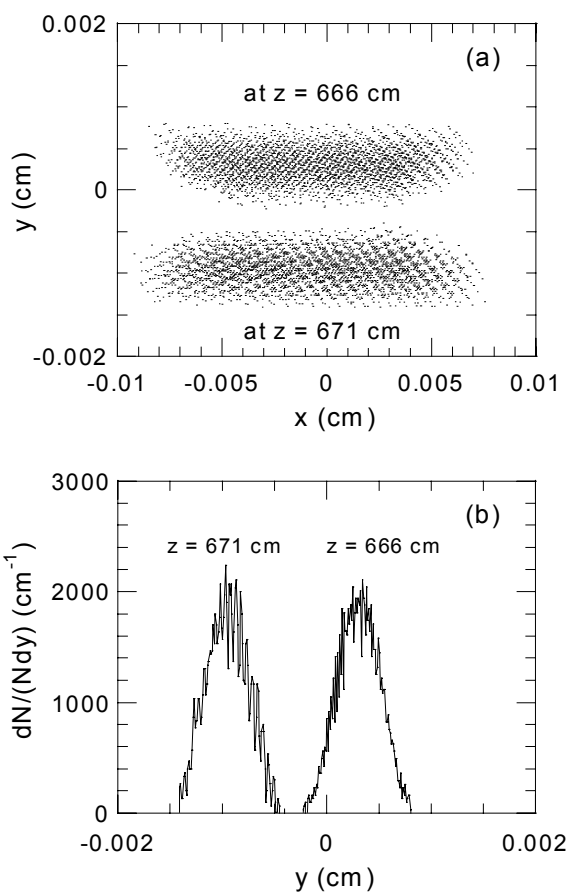
Figure 5: Sheet beam distributions $(a)$ and profiles $(b)$ following the quadrupole, at $z=666$ and $671 \mathrm{cms}$.

distributions and profiles of the bunch are shown in cross section in Fig. 5. The quadrupole magnet produces a poleface field of $1.05 \mathrm{~T}$ with a pole radius of $1 \mathrm{~cm}$, and has its axis located at $x=y=0$. The narrow transverse dimension $2 a$ of the bunch is $10 \mu \mathrm{m}$, the wide one $H$ is about $160 \mu \mathrm{m}$, and its length is $1 \mu \mathrm{m}$. These dimensions persist for a distance $>10 \mathrm{~cm}$ along the axis, which limits the length of the wake field structure.

\section{PHYSICS ISSUES}

A 2-D slab-shaped electron bunch inside a 2-D planar dielectric-slab structure has the useful property of being stable against beam break-up by transverse wakefields [7]; even if the bunch has finite height, stability can be improved [7] compared with that for a cylindrical channel with diameter $=2 a$ passing comparable charge. Thus transmission through a $10 \mu \mathrm{m}$ wide structure can be achieved over a distance of $\sim 10 \mathrm{~cm}$ so that correctly phased bunches will pick up or deposit appreciable energy in one stage. However, stability of a system of staged modules would require a focusing and transport lattice. A short bunch of charge can excite a powerful well-defined periodic train of axial electric field pulses that trail the bunch on-axis [1]. In the case of the 2-D dielectric slab structure [2], modes of the structure are free of dispersion.

The extent to which a 3-D rectangular structure of finite height, albeit with aspect ratio $\sim 20$, approaches the desirable dispersion-free quality of a pure 2-D structure, remains an object of further study. In 3-D structures, LSE and LSM are the normal modes [8]. As a structure becomes tall and narrow, the LSM modes dominate, and their dispersion relation approaches that for the 2-D slab. Thus, the frequency spacing of the LSM modes becomes nearly constant and yields a set of evenly spaced modes. Thus the wake fields set up by a finite height sheet bunch moving in a tall rectangular structure should show the clean structure similar to that found in [2]. For a train of bunches separated by $10.6 \mu \mathrm{m}$, an appropriate choice of dimensions is $2 a \sim 10 \mu \mathrm{m}$ and $b-a \sim 10 \mu \mathrm{m}$, for a dielectric constant $\varepsilon \sim 3.5$. The Coulomb field of the charge (of the same order as the peak wakefield $E_{z}$ ) following a $3.5 \mathrm{pC}$ bunch, is $\sim Q_{x} / 2 a \varepsilon_{0}$ where $Q_{x}$ is the bunch charge per unit height of the sheet bunch; this is $\sim 0.2 \mathrm{GV} / \mathrm{m}$ for just one bunch. However, the axial electric wake field may be increased further by the superposition of several following bunches, each spaced by $10.6 \mu \mathrm{m}$, and obtained by chopping the original $1 \mathrm{psec}$ bunch that was prepared by the LACARA. The breakdown field in the dielectric is determined by the $\sim 3.5$ fsec time duration for passage of a micro-bunch past a fixed region of dielectric (not by the filling time of the structure by electromagnetic energy), and therefore could exceed $3 \mathrm{GV} / \mathrm{m}$ [3].
The short dimensions of the bunch suggest that spreading along the direction of motion from the space charge field might be significant. The pulse length increment $\Delta z=z_{\text {front }}-z_{\text {back }}-L$ where $L$ is the initial bunch length, grows in time $t$ approximately as $\Delta z \approx e Q t^{2} / 2 A \varepsilon_{O} m \gamma^{3}$, where $A$ is the cross section area, $e$ and $m$ are the electron charge and rest mass, and $Q$ is the total bunch charge. The bunch lengthening effect is small on the scale of a single module, since a distance of $\sim 20 \mathrm{~m}$ is required to double the bunch length. A doubling of bunch length for $500 \mathrm{MeV}$ electrons due a beam energy spread of $0.1 \%$ requires $\sim 1 \mathrm{~km}$.

A phenomenon that arises with any physical structure where a highly relativistic electron bunch must pass through an aperture is coherent diffraction radiation [9], a form of transition radiation. In CGS units, the ratio of the energy lost by the bunch to the bunch energy $\gamma N m c^{2}$, for the rectangular slab device, is approximately

$$
\left(N e^{2} / H\right) \ln (H / a) /\left(16 \pi^{2} m c^{2}\right) \sim 10^{-5},
$$

and therefore presents no obstacle for accelerator energies in the TeV range. Each bunch in a train of $B$ bunches looses by diffraction radiation only $B^{-1}$ times the energy lost by a single bunch having the same total charge.

The wakefield structures are rigid geometrically and therefore should be reproducible and controllable elements in a staged system. Further work must be done to identify and develop broadband transparent, high dielectric constant materials suitable for these structures. Finally, the charge of a sequence of "drive" bunch charges can be programmed to increase progressively according to a certain relationship [10], thereby permitting a considerable increase in the wake field "transformer ratio".

\section{REFERENCES}

[1] S.Y. Park and J.L. Hirshfield, Phys. Rev. E 62, 1266 (2000).

[2] T-B. Zhang, J.L. Hirshfield, T.C. Marshall, and B. Hafizi, Phys. Rev. E 56, 4647 (1997).

[3] P. Sprangle, B. Hafizi, and R.F. Hubbard, Phys. Rev. E 55, 5964 (1997).

[4] S.Y. Park and J.L. Hirshfield, Phys. Plasmas 8 (2001).

[5] C. Wang and J.L. Hirshfield, Phys. Rev. E 61, 7252 (2000).

[6] W.D. Kimura et al, Phys. Rev. Lett. 86, 4041 (2001).

[7] A. Tremaine, J. Rosenzweig, and P. Schoessow, Phys. Rev. E 56, 7204 (1997).

[8] R. E. Collin, in Field Theory of Guided Waves (IEEE Press, New York, 1991).

[9] M. Castellano et al, Phys. Rev. E 63, 056501 (2001).

[10] J.G. Power, W. Gai, and A. Kanareykin, in Advanced Accelerator Concepts, $9^{\text {th }}$ Workshop, Santa Fe NM (2000) (to be publ., AIP). 